\title{
Rotational properties of Centaurs and Trans-Neptunian Objects ${ }^{\star}$
}

\section{Lightcurves and densities}

\author{
E. Dotto ${ }^{1}$, D. Perna ${ }^{1,2,3}$, M. A. Barucci ${ }^{3}$, A. Rossi ${ }^{4}$, C. de Bergh ${ }^{3}$, A. Doressoundiram ${ }^{3}$, and S. Fornasier ${ }^{3,5}$ \\ 1 INAF, Osservatorio Astronomico di Roma, via Frascati 33, 00040 Monteporzio Catone (Roma), Italy \\ e-mail: dotto@mporzio.astro.it \\ 2 Università di Tor Vergata, Roma, Italy \\ 3 LESIA, Observatoire de Paris, France \\ 4 ISTI-CNR, Pisa, Italy \\ 5 Université de Paris 7 Denis Diderot, France
}

Received 20 February 2008 / Accepted 3 May 2008

\section{ABSTRACT}

\begin{abstract}
Context. The knowledge of the rotational periods of the small bodies in the outer Solar System is a useful tool for retrieving information on the internal structure of the observed objects and for having hints on the collisional evolution state of the whole population. Aims. In order to investigate the physical nature of Centaurs and TNOs, we analysed the rotational properties of a selected sample. Methods. Photometric observations of 2 Centaurs (12929 $1999 \mathrm{TZ}_{1}$ and $956262002 \mathrm{GZ}_{32}$ ), and 5 Trans-Neptunian Objects (42355 Typhon, $479322000 \mathrm{GN}_{171}$, 65489 Ceto, $905682004 \mathrm{GV}_{9}$, and $1201322003 \mathrm{FY}_{128}$ ) were carried out with the New Technology Telescope (NTT) at the European Southern Observatory La Silla (Chile) in the framework of the ESO Large Programme 178.C-0036 (PI. M.A. Barucci).

Results. These observations allow us to estimate the rotational rate of three objects, to confirm previously published periods of two bodies, and to have the first single night lightcurves of 42355 Typhon and $1201322003 \mathrm{FY}_{128}$.

Conclusions. These data allow us to improve the available sample of determined rotational periods of TNOs and Centaurs. For 5 out of the 7 observed objects we have estimated the axis ratio $a / b$, hence the density. Our new results seem to confirm the density/dimension trend, found by Sheppard et al. (2008, in The Solar System Beyond Neptune (Univ. Arizona press), 129), with larger (brighter) objects having higher densities.
\end{abstract}

Key words. Kuiper Belt - techniques: photometric

\section{Introduction}

It is widely believed that the presently observable rotational periods of Trans-Neptunian Objects (TNOs) are the result of the collisional evolution suffered by these bodies and that the distribution of their rotational rates is related to their dimensions.

Mutual collisions contribute to modifying the angular momentum and the dimension of TNOs, therefore the distribution of their rotational rates can give us important hints on the collisional evolution state of the whole population. Most of the larger TNOs should belong to the "original" population whose rotational properties were acquired through non-disruptive collisions, while smaller objects could be fragments of originally larger bodies destroyed by catastrophic collisions (Davis \& Farinella 1997). Sheppard et al. (2008) also suggest the existence of an intermediate population of TNOs with radius between 50 and $100 \mathrm{~km}$, approximately. These objects were probably gravitationally stable to catastrophic breakup, and their present rotational properties are supposed to be strongly affected by their collisional history.

Pravec \& Harris (2000) show that the balance between the gravity force at the surface of a rotating spheroidal object, and the centrifugal force at its equator links the rotation period, the shape, and the lower limit of the density of the body. Therefore,

\footnotetext{
* Based on observations carried out at the European Southern Observatory (ESO), La Silla, Chile. ESO Large Programme 178.C0036.
}

the analysis of the rotational period of a given TNO, combined with its estimated dimension, allows us to retrieve information on the body's internal structure.

The most useful tool for investigating the rotational properties of TNOs and for computing the axis ratios of the osculating ellipsoids approximating their shapes is the analysis of photometric lightcurves. A TNO lightcurve is a function of the rotational period, the shape, and the large scale surface structure of the observed object. Therefore, analysis of photometric lightcurves allows us to retrieve rotational periods and shapes of TNOs, thereby obtaining useful information on the internal structure of each observed body and, more generally, on the rotational state of the population.

Unfortunately the presently available sample of TNO lightcurves is still rather limited. We have information on the rotational periods of about 30 TNOs and a dozen Centaurs (Sheppard et al. 2008 and references therein). In order to increase this sample, we carried out photometric observations at ESO (La Silla, Chile) of 2 Centaurs (12929 1999 TZ $_{1}$ and 95626 $2002 \mathrm{GZ}_{32}$ ) and 5 Trans-Neptunian Objects (42355 Typhon, $479322000 \mathrm{GN}_{171}, 65489$ Ceto, $905682004 \mathrm{GV}_{9}$, and 120132 $2003 \mathrm{FY}_{128}$ ), on April 2007 in the framework of the ESO Large Programme 178.C-0036 (PI. M. A. Barucci). Table 1 reports the dynamical classification by Gladman et al. (2008), the binary status, the observing dates, and the exposure time for each of the observed objects. 
Table 1. Observed objects.

\begin{tabular}{|c|c|c|c|c|}
\hline Name & Dyn. class. & $\overline{\text { Binary }}$ & Obs. date & $\begin{array}{l}\text { Exp. time } \\
\text { (s) }\end{array}$ \\
\hline $129291999 \mathrm{TZ}_{1}$ & Trojan/Centaur & No & 17-Apr-2007 & $20-60$ \\
\hline 42355 Typhon & TNO-Scattered & Yes & 18-Apr-2007 & $20-30$ \\
\hline $479322000 \mathrm{GN}_{171}$ & TNO-Resonant & No & $\begin{array}{l}\text { 19-Apr-2007 } \\
\text { 17-Apr-2007 } \\
\text { 18-Apr-2007 }\end{array}$ & $\begin{array}{c}20-60 \\
90-150 \\
25-60\end{array}$ \\
\hline 65489 Ceto & TNO-Scattered & Yes & $\begin{array}{l}\text { 17-Apr-2007 } \\
\text { 18-Apr-2007 } \\
\text { 19-Apr-2007 }\end{array}$ & $\begin{array}{c}120-150 \\
40-45 \\
60-180\end{array}$ \\
\hline $905682004 \mathrm{GV}_{9}$ & TNO-Classical & No & $\begin{array}{l}\text { 18-Apr-2007 } \\
\text { 19-Apr-2007 }\end{array}$ & $\begin{array}{c}20-25 \\
20-180\end{array}$ \\
\hline $956262002 \mathrm{GZ}_{32}$ & Centaur & No & $\begin{array}{l}\text { 17-Apr-2007 } \\
\text { 18-Apr-2007 }\end{array}$ & $\begin{array}{l}60-90 \\
20-30\end{array}$ \\
\hline $1201322003 \mathrm{FY}_{128}$ & TNO-Detached & No & $\begin{array}{l}\text { 19-Apr-2007 } \\
\text { 18-Apr-2007 } \\
\text { 19-Apr-2007 }\end{array}$ & $\begin{array}{c}20-120 \\
30-60 \\
35-180\end{array}$ \\
\hline
\end{tabular}

\section{Observations and data reduction}

The observations were performed during three nights at the $3.5 \mathrm{~m}$ New Technology Telescope (NTT) of the European Southern Observatory (La Silla, Chile), using the EMMI instrument equipped with a $2 \times 1$ mosaic of $2048 \times 4096$ MIT/LL CCD with square $15 \mu \mathrm{m}$ pixels. We used the RILD mode of EMMI for wide field imaging with the Bessell-type $R$ filter (centered at $6410 \AA$ ). The observations were carried out in a $2 \times 2$ binning mode, yielding a pixel scale of $0.33 \mathrm{arcsec} / \mathrm{pixel}$. The exposure time varied with the object magnitude and sky conditions (see Tab. 1).

The CCD images were reduced and calibrated with a standard method (see e.g. Fornasier et al. 2004), and the obtained data were corrected for lightime. The instrumental magnitudes were measured using aperture photometry with an integrating diameter typically about three times the average seeing, and sky subtraction was performed using a 5-10 pixel wide annulus. Since the sky conditions were not photometric, we performed differential photometry without absolute calibration, by using at least three field stars for each observed object. To minimize the random errors in the measurements, the selected field stars were chosen so as to be brighter than the target. From the visual inspection and the radial profile analysis of the images, no coma was detected for any of the observed objects.

The obtained single night lightcurves are shown in Fig. 1. The error bars are based on the photometric errors and the instrumental magnitude statistics.

\section{Results}

The rotational synodic periods were computed by applying the method based on the Fourier analysis of the lightcurves, developed by Harris et al. (1989). The obtained composite lightcurves are shown in Figs. 2-6.

For 5 out of the 7 observed objects, we derived a lower limit of the axis ratio $a / b$, supposing that they do not have considerable albedo variations and that the observed lightcurve amplitudes are only affected by the elongated shapes. We considered that, for an ellipsoidal object with axes $a>b>c$ observed at an aspect angle $\xi$, the lightcurve amplitude $\Delta m$ is given by (Binzel et al. 1989)

$\Delta m=2.5 \log \left(\frac{a}{b}\right)-1.25 \log \left(\frac{a^{2} \cos ^{2} \xi+c^{2} \sin ^{2} \xi}{b^{2} \cos ^{2} \xi+c^{2} \sin ^{2} \xi}\right)$ where the maximum value of

$\Delta m=2.5 \log \left(\frac{a}{b}\right)$

corresponds to the equatorial view $\left(\xi=90^{\circ}\right)$.

The results obtained for each target are discussed below.

$129291999 \mathbf{T Z}_{1}$ orbits around the Sun in the region of the Jupiter Trojans. At the time of our observations, it was classified as a Centaur. However, recent dynamical integrations seem to suggest it is a typical Trojan (Moullet et al. 2008). It has an absolute magnitude $H=9.3$. We observed this object during one night for more than $7 \mathrm{~h}$. The lightcurve amplitude is at least of $0.15 \pm 0.02 \mathrm{mag}$, hence the axis ratio $a / b$ is at least $1.15 \pm 0.02$. The data seem to suggest a single peaked periodicity of about $5 \mathrm{~h}$, in good agreement with the double peaked rotational period of $10.4 \mathrm{~h}$ suggested by Moullet et al. (2008) (Fig. 2).

42355 Typhon is classified as a scattered object. Recent observations have shown the binary nature of this body (Noll et al. 2006). Its absolute magnitude is $H=7.2$. We observed this object during 2 nights for more than $8 \mathrm{~h}$. The data reported in Fig. 1 did not show any peculiar periodicity and were not enough to determine the rotational period, which must be longer than the $5 \mathrm{~h}$ of continuous observation performed during each observing night.

$479322000 \mathbf{G N}_{171}$ is a resonant TNO already observed by Sheppard and Jewitt (2002), who obtained a double peaked composite lightcurve with a rotational period of $8.329 \pm 0.005 \mathrm{~h}$. Our observations, carried out during two different observing nights, confirm this period. The composite lightcurve (Fig. 3) presents a large amplitude of $0.60 \pm 0.03$, which gives an axis ratio $a / b \geq 1.74 \pm 0.04 .2000 \mathrm{GN}_{171}$ is therefore one of the most elongated TNOs.

65489 Ceto, classified as a scattered object, has an absolute magnitude of 6.3. We observed this object during three different nights for a total of about $14 \mathrm{~h}$. The results, shown in Fig. 4, suggest a double peaked rotational period of $4.43 \pm 0.03 \mathrm{~h}$. The lightcurve amplitude is of about $0.13 \pm 0.02$, hence the lower limit of the axis ratio $a / b$ is $1.13 \pm 0.02$. On the basis of this result, 65489 Ceto is the second fastest rotator among TNOs after $1361082003 \mathrm{EL}_{61}$. This is a very unexpected result, because Grundy et al. (2007), through Hubble Space Telescope observations, have revealed that this object is a close binary system, 

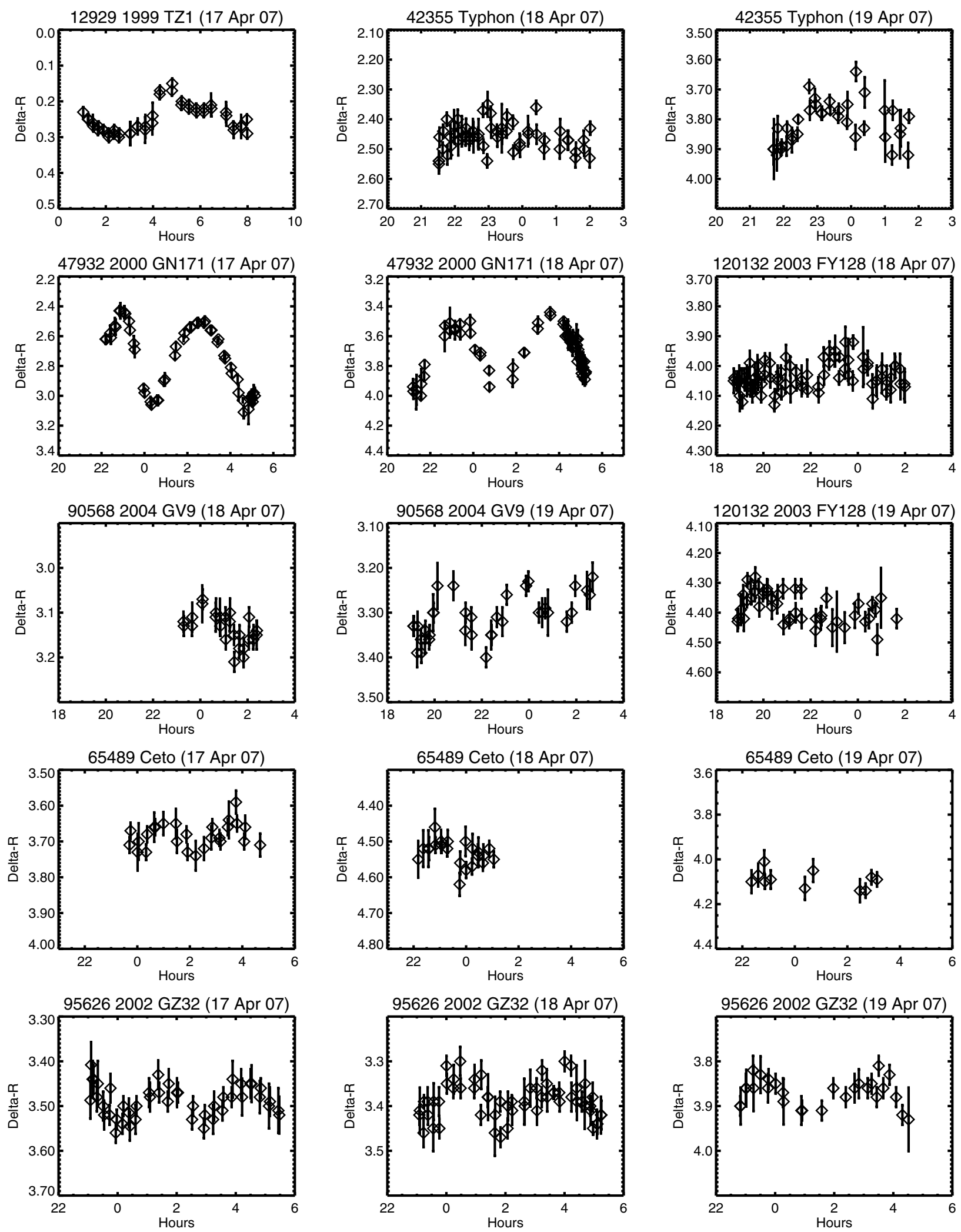

Fig. 1. Single night lightcurves of the observed objects.

composed of two components of about 87 and $66 \mathrm{~km}$, with a bulk density of about $1.37 \mathrm{~g} / \mathrm{cm}^{3}$. In particular, our derived rotation period implies that the system is clearly not completely spin locked (as for example the Pluto-Charon system), the rotation rate of the primary being much faster than the orbital period of the binary system (estimated at about 9.55 days by Grundy et al.). The total angular momentum (orbital plus rotational) of the binary system is slightly in excess of the critical one for rotational fission (e.g., Descamps \& Marchis 2008). These facts might suggest a recent tidal breakup origin for the Ceto/Phorcys system.

$905682004 \mathrm{GV}_{9}$ is a classical TNO with absolute magnitude $H=4$. We observed this object during 2 nights for more than 


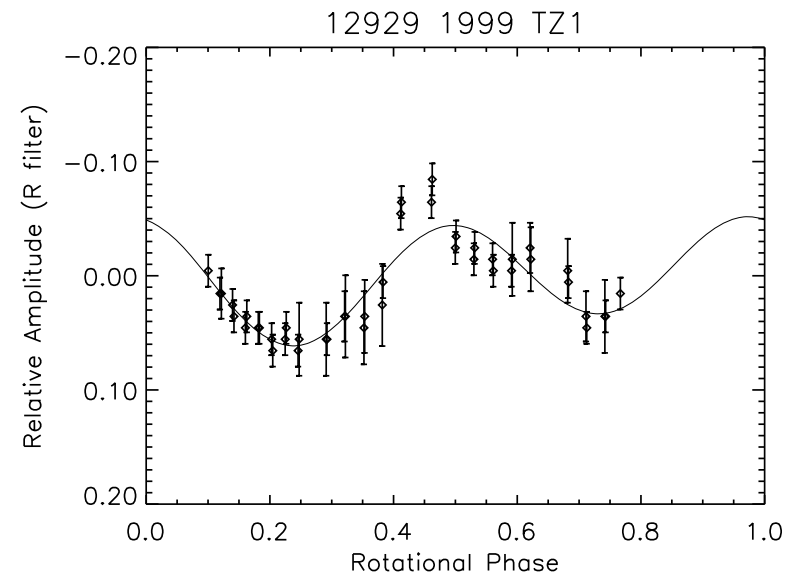

Fig. 2. Composite lightcurve of $129291999 \mathrm{TZ}_{1}$ obtained with $P_{\text {syn }}=$ $10.4 \mathrm{~h}$. The lightcurve zero point is at 0 UT on 2007 April 17th.

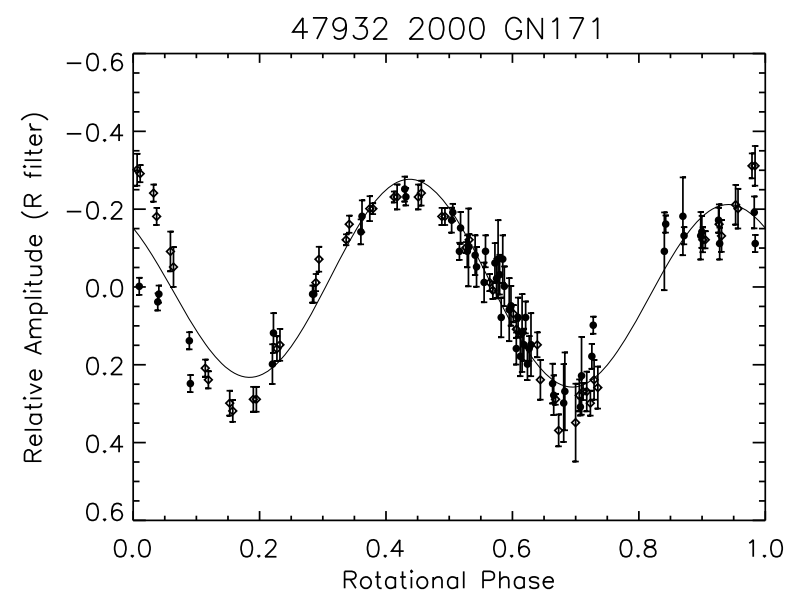

Fig. 3. Composite lightcurve of $479322000 \mathrm{GN}_{171}$ obtained with $P_{\text {syn }}=$ 8.329 h. The lightcurve zero point is at 0 UT on 2007 April 18th.

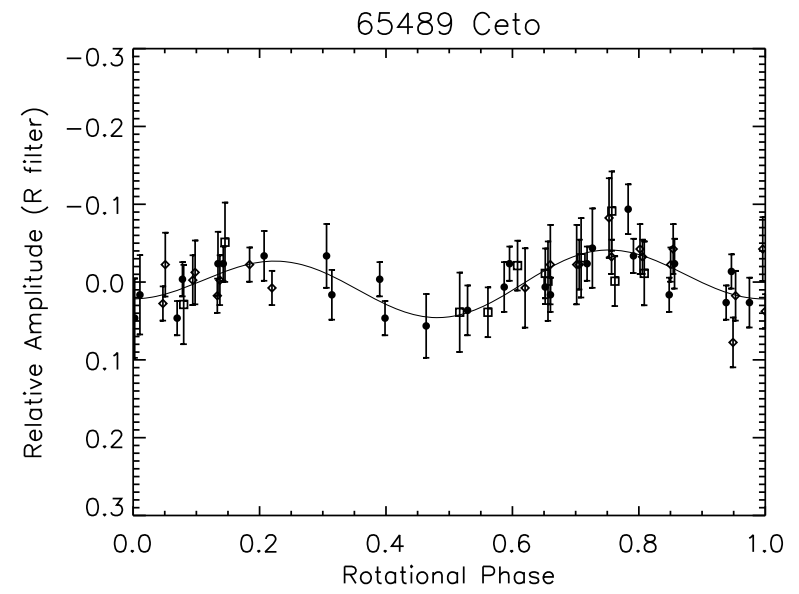

Fig. 4. Composite lightcurve of 65489 Ceto obtained with $P_{\text {syn }}=4.43 \mathrm{~h}$. The lightcurve zero point is at 0 UT on 2007 April 18th.

11 h. The composite lightcurve shown in Fig. 5 has been obtained with a rotational period of $5.86 \pm 0.03 \mathrm{~h}$. The lightcurve amplitude of $0.16 \pm 0.03$ gives $a / b \geq 1.16 \pm 0.03$.

$956262002 \mathbf{G Z}_{32}$ is a Centaur. We observed this object during three different nights for more than $18 \mathrm{~h}$. The double peaked composite lightcurve shown in Fig. 6 has been obtained with a

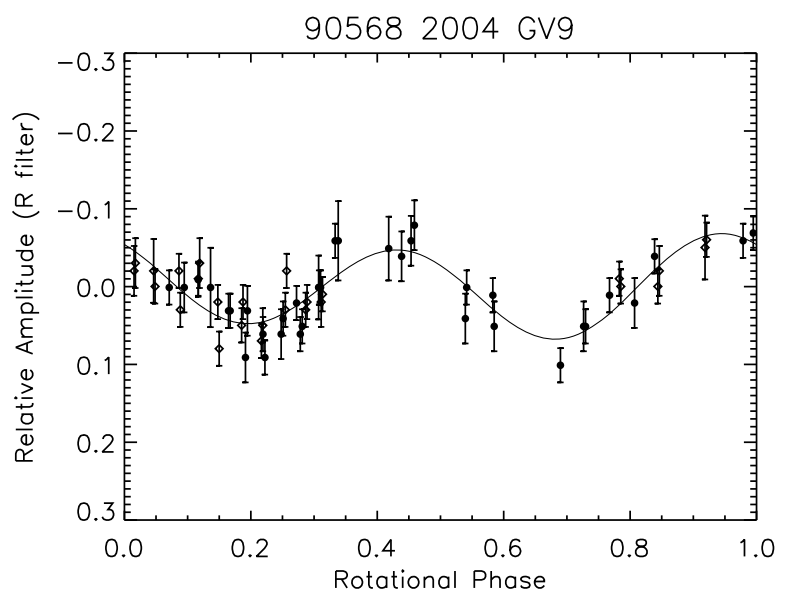

Fig. 5. Composite lightcurve of $905682004 \mathrm{GV}_{9}$ obtained with $P_{\text {syn }}=$ $5.86 \mathrm{~h}$. The lightcurve zero point is at 0 UT on 2007 April 19th.

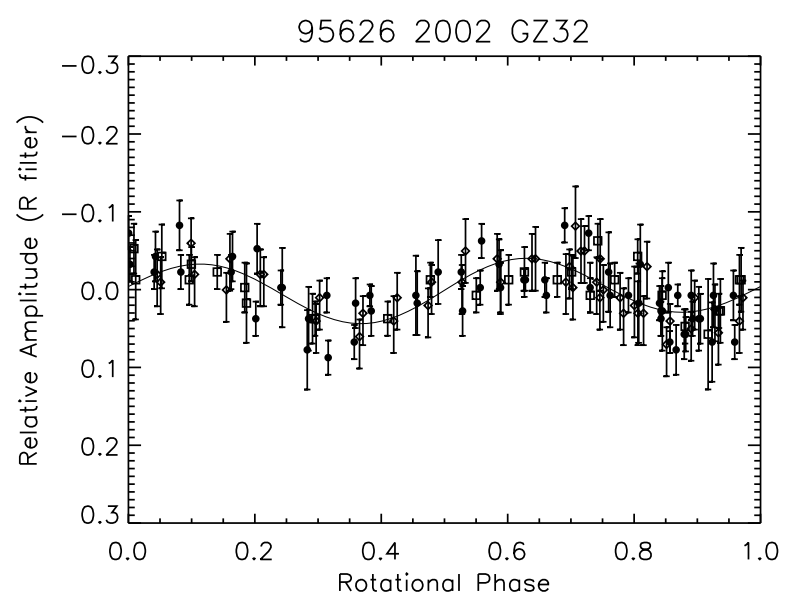

Fig. 6. Composite lightcurve of $956262002 \mathrm{GZ}_{32}$ obtained with $P_{\text {syn }}=$ $5.80 \mathrm{~h}$. The lightcurve zero point is at 0 UT on 2007 April 18th.

synodic period of $5.80 \pm 0.03 \mathrm{~h}$. The lightcurve amplitude of $0.15 \pm 0.03$ suggests an axis ratio $a / b$ more than $1.15 \pm 0.03$.

1201322003 FY $_{128}$ is a detached TNO observed in the present survey during 2 different nights for more than $13 \mathrm{~h}$. The single night lightcurves shown in Fig. 1 did not allow determination of the rotational period of this object, which is likely longer than the $7 \mathrm{~h}$ of continuous observation performed during the first night.

\section{Discussion}

From the obtained synodic periods $\left(P_{\text {syn }}\right)$ and the computed lightcurve amplitudes $(\Delta m)$, by applying the equation by Pravec $\&$ Harris (2000) for the density of an object rotating with angular velocity $2 \pi / P_{\text {syn }}$, we estimated the lower limit for the density of 4 out of the 7 observed objects as:

$\rho=\left(\frac{3.3}{P_{\text {syn }}}\right)^{2}(1+\Delta m)$.

For $479322000 \mathrm{GN}_{171}$, due to its large lightcurve amplitude, we adopted the contact spheres' model, proposed by Jewitt \& Sheppard (2002) for 20000 Varuna, which estimates the density as:

$\rho=\frac{3 \pi}{G P_{\text {syn }}^{2}} \frac{\left[1+\left(10^{0.4 \Delta m}-1\right)^{1 / 2}\right]^{3}}{1+\left(10^{0.4 \Delta m}-1\right)^{3 / 2}}$ 
Table 2. Obtained results.

\begin{tabular}{|c|c|c|c|c|c|c|}
\hline$\overline{\overline{\text { Name }}}$ & $\overline{\mathrm{H}}$ & $\begin{array}{c}\text { Rot. Period } \\
\text { (hours) } \\
\end{array}$ & $\begin{array}{l}\Delta m \\
\text { mag } \\
\end{array}$ & $\overline{\mathrm{a} / \mathrm{b}}$ & $\begin{array}{c}\rho \\
\mathrm{g} / \mathrm{cm}^{3}\end{array}$ & $\begin{array}{c}\rho \\
\text { Jacobi ell. } \\
\mathrm{g} / \mathrm{cm}^{3} \\
\end{array}$ \\
\hline $129291999 \mathrm{TZ}_{1}$ & 9.3 & $10.4^{1}$ & $0.15 \pm 0.02$ & $1.15 \pm 0.02$ & $0.11^{3}$ & $0.36-0.47$ \\
\hline 42355 Typhon & 7.2 & $>5$ & & & & \\
\hline $479322000 \mathrm{GN}_{171}$ & 6.0 & $8.329 \pm 0.005^{2}$ & $0.60 \pm 0.03$ & $1.74 \pm 0.04$ & $0.62^{4}$ & $0.56-0.74$ \\
\hline 65489 Ceto & 6.3 & $4.43 \pm 0.03$ & $0.13 \pm 0.02$ & $1.13 \pm 0.02$ & $0.63^{3}$ & $1.99-2.61$ \\
\hline $905682004 \mathrm{GV}_{9}$ & 4.0 & $5.86 \pm 0.03$ & $0.16 \pm 0.03$ & $1.16 \pm 0.03$ & $0.37^{3}$ & $1.14-1.49$ \\
\hline $956262002 \mathrm{GZ}_{32}$ & 6.8 & $5.80 \pm 0.03$ & $0.15 \pm 0.03$ & $1.15 \pm 0.03$ & $0.37^{3}$ & $1.16-1.52$ \\
\hline $1201322003 \mathrm{FY}_{128}$ & 5.0 & $>7$ & & & & \\
\hline
\end{tabular}

${ }^{1}$ By Moullet et al. (2008); ${ }^{2}$ by Sheppard \& Jewitt (2002); ${ }^{3}$ by Eq. (3); ${ }^{4}$ by Eq. (4).

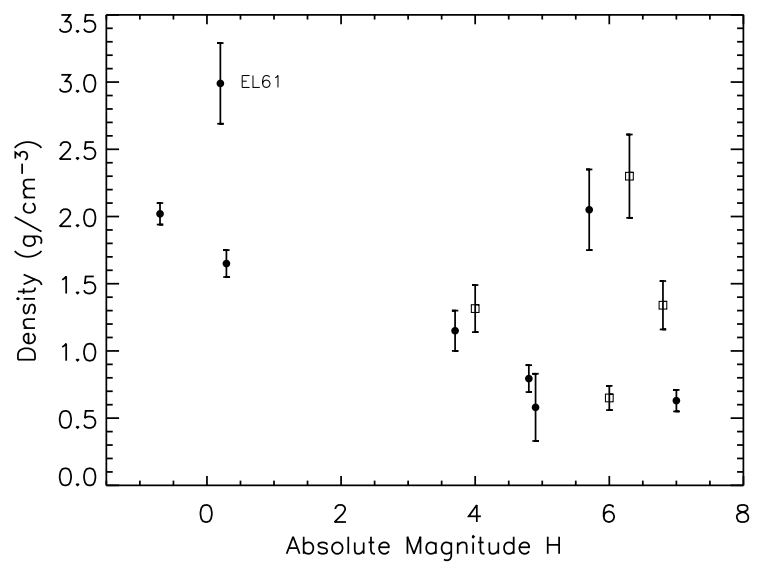

Fig. 7. Estimated densities of TNOs vs. their absolute magnitudes. Filled circles show the densities of Pluto, Charon, Varuna, $2003 \mathrm{EL}_{61}$, $1999 \mathrm{TC}_{36}, 2001 \mathrm{QG}_{298}, 2001 \mathrm{CZ}_{31}, 1995 \mathrm{SM}_{55}$ taken from Sheppard et al. (2008) and references therein. Open squares show the density ranges reported in the last column of Table 2.

where $G$ is the gravitational constant. With both these models, the lower limit densities have been computed from the observed periods and lightcurve amplitudes, throughout the lower limit of the axis ratio given by Eq. (2).

An alternative estimation of the density lower limit was obtained from rotational periods and lightcurve amplitudes (and hence from the lower limit of the axis ratio $a / b$ ) following the Chandrasekhar (1987) table for rotational stable Jacobi ellipsoids. Considering that ellipsoids with $a / b>2.31$ are unstable due to the effect of the rotation (Jeans 1919), a higher limiting density was obtained from the same table by imposing $a / b=2.31$.

Table 2 lists the absolute magnitude, taken from the MPC, of each observed object, the computed rotational period, the composite lightcurve amplitude, the lower limit of the axis ratio $a / b$, the lower limit of the density obtained with Eq. (3), or Eq. (4) for $479322000 \mathrm{GN}_{171}$, and the range of variation in the density as given by the Chandrasekhar (1987) table for Jacobi ellipsoids.

Following Sheppard et al. (2008), Fig. 7 shows the estimated densities vs. the absolute magnitudes, for TNOs with absolute magnitude $H<7$ (hence $D \geq 200 \mathrm{~km}$ with moderate albedo). Although different models gave for our targets different estimations of the density, for no object we reach density values as high as $3 \mathrm{~g} / \mathrm{cm}^{3}$. The data so far available seem to confirm the dimension/density trend found by Sheppard et al. (2008), with larger (brighter) objects denser than the smaller (fainter) ones. As shown in Fig. 7 this trend seems to be strongly triggered by the extremely high density of $1361082003 \mathrm{EL}_{61}$, therefore more data are absolutely needed to confirm or refute it. As suggested by Sheppard et al. (2008), if confirmed this could be due to the porosity of the objects of different sizes and/or to the mass fraction of rocks vs. ices.

\section{Conclusion}

On April 2007 at ESO-NTT, we performed photometric observations of 7 objects, among Centaurs, TNOs and scattered objects. The results allowed us

1. to compute the rotational period of three objects (65489 Ceto, $905682004 \mathrm{GV}_{9}$, and $956262002 \mathrm{GZ}_{32}$ ) and to confirm the rotational periods of $129291999 \mathrm{TZ}_{1}$ and 47932 $2000 \mathrm{GN}_{171}$;

2. to compute the density and to estimate a lower limit for the axis ratio $a / b$ of 5 out of the 7 observed bodies. Three of the rotational periods we computed are quite short for TNOs, but the computed lower limit of the density of each object is low enough to affirm that the rotational parameters we found are reasonable for rotationally stable ellipsoids;

3. to confirm the dimension/density trend found by Sheppard et al. (2008), with larger (brighter) objects denser than the smaller (fainter) ones.

\section{References}

Binzel, R. P., Farinella, P., Zappalà, V., et al. 1989 in Asteroids II (Univ. of Arizona press), 415

Chandrasekhar, S. 1987, Ellipsoidal Figures of Equilibrium (New York: Dover) Davis, D., \& Farinella, P. 1997, Icarus 125, 50

Descamps, P., \& Marchis, F. 2088, Icarus 193, 74

Fornasier, S., Doressoundiram, A., Tozzi, G. P., et al. 2004, A\&A, 421, 353

Gladman, B., Marsden, B. G., \& Van Laerhoven C. 2008, in The Solar System Beyond Neptune (Univ. of Arizona press), 43

Grundy, W. M., Stansberry, J. A., Noll, K. S., et al. 2007, Icarus, 191, 286

Harris, A. W., Young, J., Bowell, E., et al. 1989, Icarus, 77, 171

Jeans, J. 1919, Problems of cosmogony and stellar dynamics (London/New York: Cambridge Univ. Press)

Jewitt, D., \& Sheppard, S. S. 2002, AJ., 123, 2110

Moullet, A., Lellouch, E., Doressoundiram, A., et al. 2008, A\&A, 483, 17 Noll, K. S., Grundy, W. M., Stephens, D. C., et al. 2006, IAU Circ., 8689, 1

Pravec, P., \& Harris, A. W. 2000, Icarus, 148, 12

Sheppard, S. S., \& Jewitt, D. 2002, AJ., 124, 1757

Sheppard, S. S., Lacerda, P., \& Ortiz, J. L. 2008, in The Solar System Beyond

Neptune (Univ. of Arizona press), 129 